\title{
Genetics and functional genetics of autoimmune diseases
}

\author{
Yukinori Okada ${ }^{1,2} \cdot K_{\text {Kazuhiko Yamamoto }}^{3}$
}

Published online: 10 February 2022

(c) The Author(s), under exclusive licence to Springer-Verlag GmbH Germany, part of Springer Nature 2022

The current issue of seminars in immunopathology "Genetics and functional genetics of Autoimmune diseases" presents a number of review articles describing current and future landscape of human genetic studies in the field of autoimmune diseases. Immunological phenomena related to self-defense and disease are caused by the interaction of many molecules in different types of immunocompetent cells. Immunological research has been actively conducted in animal models, mainly mice. The development of analytical methodologies, including gene knockout, has provided us with new findings. However, although the mouse and human immune systems are similar in principle, there are differences in detail. Treatments that are effective in mouse models of immune diseases are not always effective in humans. Therefore, the importance of research that directly targets the human immune system has been proposed.

The cause and outcome cannot be clarified from the observation of phenomena in the immune response alone. It is difficult to determine whether observed gene expression, protein expression, and epigenetics data are the cause or the consequence of a disease. Therefore, an approach to clearly distinguish cause from outcome, such as gene knockout in mice, would be necessary for human immunology. Genetic variants of susceptibility to disease deserve attention to achieve this goal. With several exceptions, such as

This article is a contribution to the special issue on: Genetics and functional genetics of Autoimmune diseases - Guest Editors: Yukinori Okada and Kazuhiko Yamamoto

Yukinori Okada

yokada@sg.med.osaka-u.ac.jp

$\triangle$ Kazuhiko Yamamoto

kazuhiko.yamamoto@ riken.jp

1 Laboratory for Systems Genetics, RIKEN Center for Integrative Medical Sciences, Yokohama, Japan

2 Department of Statistical Genetics, Osaka University Graduate School of Medicine, Suita, Japan

3 Laboratory of Autoimmune Diseases, RIKEN Center for Integrative Medical Sciences, Yokohama, Japan antigen receptor genes in $\mathrm{T}$ and $\mathrm{B}$ cells, genomic information exists before the onset of the disease and does not change. Genetic variants that are significantly associated with each phenomenon of a disease provide a clear link to the cause of that phenomenon.

With the initial decoding of the whole human genome in 2003 , discussions began on the feasibility of analyzing disease-susceptible genetic variants in common diseases (i.e., multifactorial or polygenic diseases), which includes autoimmune diseases. Among human genome variant categories, single nucleotide polymorphism (SNP) has been the main focus. The international HapMap project has constructed a catalog of genome-wide haplotype (linkage disequilibrium; LD) blocks and tag SNPs [1]. Then, along with the development of microarray SNP typing technology, the current genome-wide association study (GWAS) methodology was established. To date, GWAS has been conducted for more than a thousand of human complex traits including many immune-related phenotypes such as autoimmune diseases, providing a massive list of disease risk variants [2, 3].

In contrast to rare Mendelian diseases where risk variants are mostly altering protein sequences, majority of the GWAS-reported risk genetic variants are located in non-coding regions of human genome, typically found to be expression quantitative trait loci (eQTLs) that have dosage effects on gene expression levels. The eQTL is a key phenomenon for regulating gene expression in human cells, and can be further characterized to be specific to cell types. This cell specificity is largely due to epigenetic changes, such as celltype-specific histone modifications and DNA methylation, in specific regions of chromosomes. Disease susceptibility variants overlap significantly with the location of histone modifications in promoters and active enhancers of specific immune cell subsets [4]. Therefore, causal information can be extracted by measuring the functional molecules of immunocompetent cells by focusing on disease-susceptible genetic variants, gene expression (mRNA), epigenome, and proteins. An integrated understanding of such endophenotypes will enable the development of functional genetics that provide a detailed understanding of an individual's immune 
status, including causal and consequential factors [5]. Currently, the development of functional genetics is being rapidly promoted.

In this special issue, we would like to review the current status of genetic analyses and functional genetics of autoimmune diseases and discuss the next generation of autoimmune research. The review by Ishigaki et al. [6] provides the current landscape and future directions of GWAS. How to connect simple case-control associations of the genetic variants with functional genomics resources is important to understand disease genetics and biology. Human leukocyte antigen (HLA) genes at 6p23 explain significant proportions of genetic risk of autoimmune diseases. Naito et al. [7] presents current fine-mapping efforts to dissect causal HLA variants of autoimmune diseases. Then, Ha et al. [8], Padyukov et al. [9], Kim et al. [10], and Márquez et al. [11] summarize recent achievements in genetics of four major autoimmune diseases of systemic lupus erythematosus, rheumatoid arthritis, multiple sclerosis, and type 1 diabetes, respectively. More than a hundred of risk variants have been massively identified for these autoimmune diseases through active international collaboration networks.

Utilization of functional genomic resources by the latest technologies is essential to interpret functional impacts of the disease risk variants. Wang et al. [12] reviews statistical methods to fine-map causal variants from GWAS findings, as well as empirical application examples in autoimmune disease genetics. Orozco et al. [13] introduces fine-mapping efforts based on a set of epigenetic information including high dimensional chromatin structure in cells. Guerrini et al. [14] describes a framework to utilize cap analysis of gene expression (CAGE) to reveal noncoding regulatory elements in the human genome sequences. To validate biological impacts of the fine-mapped variants, functional interrogation of the candidate variants is useful. Ding et al. [15] discusses two main interrogation methods of CRISPR/Cas9 technologies and massively parallel reporter assays.

We sincerely hope a series of the contents in this issue will provide novel insights into genetics and functional genetics of autoimmune diseases.

\section{References}

1. International HapMap Consortium (2005) A haplotype map of the human genome. Nature 437:1299-1320
2. Buniello A et al (2019) The NHGRI-EBI GWAS catalog of published genome-wide association studies, targeted arrays and summary statistics nucleic. Acids Res. 47:D1005-D1012

3. Okada Y, Eyre S, Suzuki A, Kochi Y, Yamamoto K (2019) Genetics of rheumatoid arthritis: 2018 status. Ann Rheum Dis 78:446-453

4. Trynka G et al (2013) Chromatin marks identify critical cell types for fine mapping complex trait variants. Nat Genet 45:124-130

5. Amariuta T, Luo Y, Knevel R, Okada Y, Raychaudhuri S (2020) Advances in genetics toward identifying pathogenic cell states of rheumatoid arthritis. Immunol Rev 294:188-204

6. Ishigaki K (2022) Beyond GWAS: from simple associations to functional insights. Semin Immunpathol. https://doi.org/10.1007/ s00281-021-00894-5

7. Naito T, Okada Y (2022) HLA imputation and its application to genetic and molecular fine-mapping of the MHC region in autoimmune diseases. Semin Immunpathol. https://doi.org/10.1007/ s00281-021-00901-9

8. Ha E, Bae SC, Kim K (2022) Recent advances in understanding the genetic basis of systemic lupus erythematosus. Semin Immunpathol. https://doi.org/10.1007/s00281-021-00900-w

9. Padyukov L (2022) Genetics of rheumatoid arthritis. Semin Immunpathol. https://doi.org/10.1007/s00281-022-00912-0

10. Kim W, Patsopoulos NA (2022) Genetics and functional genomics of multiple sclerosis. Semin Immunpathol. https://doi.org/10. 1007/s00281-021-00907-3

11. Márquez A, Martín J (2022) Genetic overlap between type 1 diabetes and other autoimmune diseases. Semin Immunpathol. https://doi.org/10.1007/s00281-021-00885-6

12. Wang Q, Huang H (2022) Methods for statistical fine-mapping and their applications to auto-immune diseases. Semin Immunpathol. https://doi.org/10.1007/s00281-021-00902-8

13. Orozco G (2022) Fine mapping with epigenetic information and 3D structure. Semin Immunpathol. https://doi.org/10.1007/ s00281-021-00906-4

14. Guerrini MM, Oguchi A, Suzuki A, Murakawa Y (2022) Cap analysis of gene expression (CAGE) and noncoding regulatory elements. Semin Immunpathol. https://doi.org/10.1007/ s00281-021-00886-5

15. Ding J, Frantzeskos A, Orozco G (2022) Functional interrogation of autoimmune disease genetics using CRISPR/Cas9 technologies and massively parallel reporter assays. Semin Immunpathol. https://doi.org/10.1007/s00281-021-00887-4

Publisher's note Springer Nature remains neutral with regard to jurisdictional claims in published maps and institutional affiliations. 